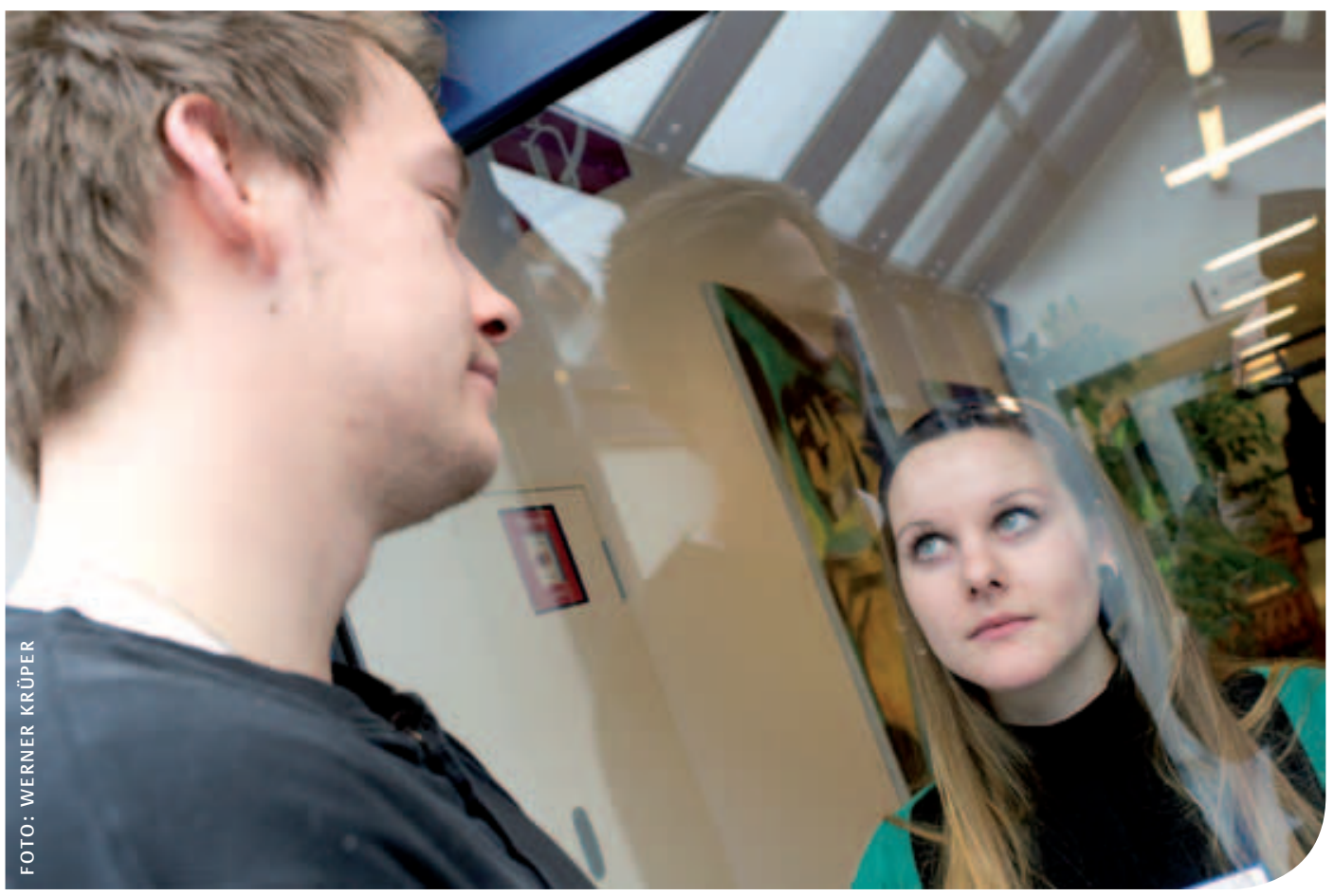

KOMMUNIKATION IN DER STATIONÄREN AKUTPSYCHIATRIE

\title{
„Ich habe mir überlegt, was Ihnen wohl am meisten weh tut"
}

\author{
Elke Prestin, Michael Schulz
}

Wenn eine Sprachwissenschaftlerin, die einige Monate in einer psychiatrischen Akutklinik verbracht hat, mit einem Pflegewissenschaftler über Kommunikation in der Psychiatrie redet, ist das spannend. So spannend, dass es lohnt, ein breiteres Publikum mit einzubeziehen. Mit einer kritisch-konstruktiven Analyse möchten wir zu einer Diskussion darüber anregen, welche Botschaften professionelle Pflegekräfte direkt und indirekt an Patienten vermitteln und welche Haltungen sich dahinter verbergen. Im Mittelpunkt stehen die Erfahrungen, welche die Erstautorin als Patientin gemacht hat. Um diese systematisch und wissenschaftlich fundiert auswerten zu können, nutzen wir Modelle und Methoden aus der Kommunikationssoziologie. Zudem wird an einzelnen Stellen auf Parallelen zur Pflegewissenschaft Bezug genommen. Für eher wissenschaftsferne Leserinnen und Leser mag die alleinige Lektüre der „Beobachtungen aus der Akutpsychiatrie“ im zweiten Teil dieses Beitrags (ab Seite 89) genügen, denn hier geht es um den konkreten Praxisbezug. Wer jedoch an der theoretischen Fundierung pflegerischen Handelns interessiert ist, dem sei der gesamte Aufsatz empfohlen.

Nicht auf Sand gebaut - der wissenschaftliche Hintergrund

Den theoretischen Hintergrund der folgenden Ausführungen bildet das Konzept der Kontextualisierung. Dieser sozialkonstruktivistische Ansatz befasst sich mit der Frage, wie durch Kommunikation Wirklichkeit geschaffen und verändert wird. Der
Begriff der Kontextualisierung wurde von Jenny Cook-Gumperz und John Gumperz [5] geprägt. Zentral ist die Annahme, dass Kontext und Kommunikation einander wechselseitig beeinflussen: Einerseits prägen die jeweiligen sozialen und kulturellen Rahmenbedingungen den Sprachgebrauch. Zugleich werden Strukturen und Normen einer Gesellschaft aber durch Kommunikation überhaupt erst geschaffen und aufrecht erhalten. Was dies auf gesellschaftlicher Ebene bedeutet, ist Gegenstand der Ethnografie der Kommunikation [vgl. 13]. John Gumperz [12] dagegen bezieht sich in seiner interaktionalen Soziolinguistik auf einzelne, konkrete Kommunikations- 
situationen. Auch hier gilt demnach: Der Kontext ist keine feststehende äußere Einflussgröße, sondern er wird interaktiv produziert.

Zur Veranschaulichung sei auf ein zufälliges Treffen zwischen einem Arzt und einem Patienten auf dem Klinikflur verwiesen. Zwar haben beide Gesprächspartner gesellschaftlich definierte Rollen inne, doch ob sie in der aktuellen Situation innerhalb dieser Rollen agieren, entscheiden sie selbst. So ist vorstellbar, dass der Arzt - entsprechend der Rolle „Arzt“ - eine professionelle Befindensfrage stellt, etwa die, ob der Patient ein bestimmtes Medikament inzwischen besser vertrage. Er kann aber auch mit der Frage „Na, was tippen Sie denn für heute Abend?" auf ein anstehendes Fußball-Länderspiel Bezug nehmen. Wenn der Patient einen Tipp abgibt und dann seinerseits den Arzt nach seiner Vorhersage fragt, agieren beide in der Rolle als „Fußball-Interessierte“, die nicht hierarchisch strukturiert ist.

Dass die letztgenannte Variante in der Praxis äußerst selten anzutreffen ist, dürfte zwei Gründe haben: Zum einen gilt es bis heute vielfach als Zeichen von Professionalität, wenn Mitarbeitende in Gesundheitsberufen sich am Arbeitsplatz ausschließlich innerhalb ihrer beruflichen Rolle bewegen. Der zweite Grund dafür, dass Kommunikationsmuster außerhalb der Rollen „Arzt“ und „Patient“ bzw. „Pflegekraft“ und „Patient“ in Kliniken kaum vorkommen, liegt sicherlich darin, dass das dafür notwendige Wissen über den Gesprächspartner gar nicht vorhanden ist. Gerade in der Psychiatrie fehlen damit jedoch Anknüpfungspunkte nicht nur für Alltagskommunikation, sondern auch für ein tieferes Verständnis des Gegenübers und für individuelle therapeutische Strategien. Denn was einen Menschen wirklich bewegt, was ihm wichtig ist, was seinen ganz persönlichen Lebenssinn ausmacht, lässt sich eben nur im persönlichen Gespräch erfahren und erspüren. Das aber ist außerhalb klassischer Rollenmuster wesentlich einfacher, vielleicht sogar überhaupt erst möglich. Alltagskommunikation, auch wenn sie zunächst nur ganz oberflächlich sein mag wie im genannten Beispiel, kann hier als wesentlicher „Türöffner“ wirken.

Wenn man nun davon ausgeht, dass der Kontext erst durch die Kommunikation hervorgebracht wird, dann stellt sich als Nächstes die Frage, wie das genau geschieht. Warum also agiert jemand erkennbar in der Rolle als Arzt, wenn er nach dem Befinden des Patienten fragt, aber als Fußballfan, wenn er um einen Tipp für das Länderspiel bittet? Gumperz [11] spricht in diesem Zusammenhang von Kontextualisierungshinweisen („Contextualisation cues“). Bestimmte Elemente der Kommunikation, beispielsweise das Wort „Tipp“, vielleicht auch Tonfall und Körpersprache, ver-

hingegen auf dem Flur statt, dann fällt eine typische Komponente, nämlich das Sprechzimmer, schon einmal weg. Wenn dann der Arzt auch noch über Fußball statt über Medizin spricht, ist die ursprüngliche Rahmenvorstellung nicht mehr haltbar. Stattdessen wird ein neuer Rahmen herangezogen, nämlich der, welcher am besten mit der aktuellen Situation übereinstimmt (im genannten Beispiel: der Rahmen einer privaten Kommunikation, hier eines lockeren Gespräches unter Fußballinteressierten).

Wir haben hier zunächst von „Rahmen“ gesprochen, weil dieser auf Erving Goffman [9] zurückgehende Begriff auch von Gumperz in seinem Kontextualisierungsansatz verwendet wurde. Den Zusammenhang von Kontextualisierungshinweisen und Rahmen fasst Uta Quasthoff [16, S. 25]

weisen auf den Rahmen, innerhalb dessen das kommunikative Handeln zu deuten ist. Den Hintergrund dafür bildet die Annahme, dass die Beteiligten an einer Kommunikationssituation laufend ihr aktuelles Erleben mit bestimmten erlernten Erwartungsmustern abgleichen. Dazu gehört beispielsweise die Erwartung, dass eine Ärztin im (Visitenoder Therapie-)Gespräch krankheits- und therapiebezogene Fragen stellt, in ihren Unterlagen blättert und sich Notizen macht. Findet die Arzt-Patienten-Kommunikation

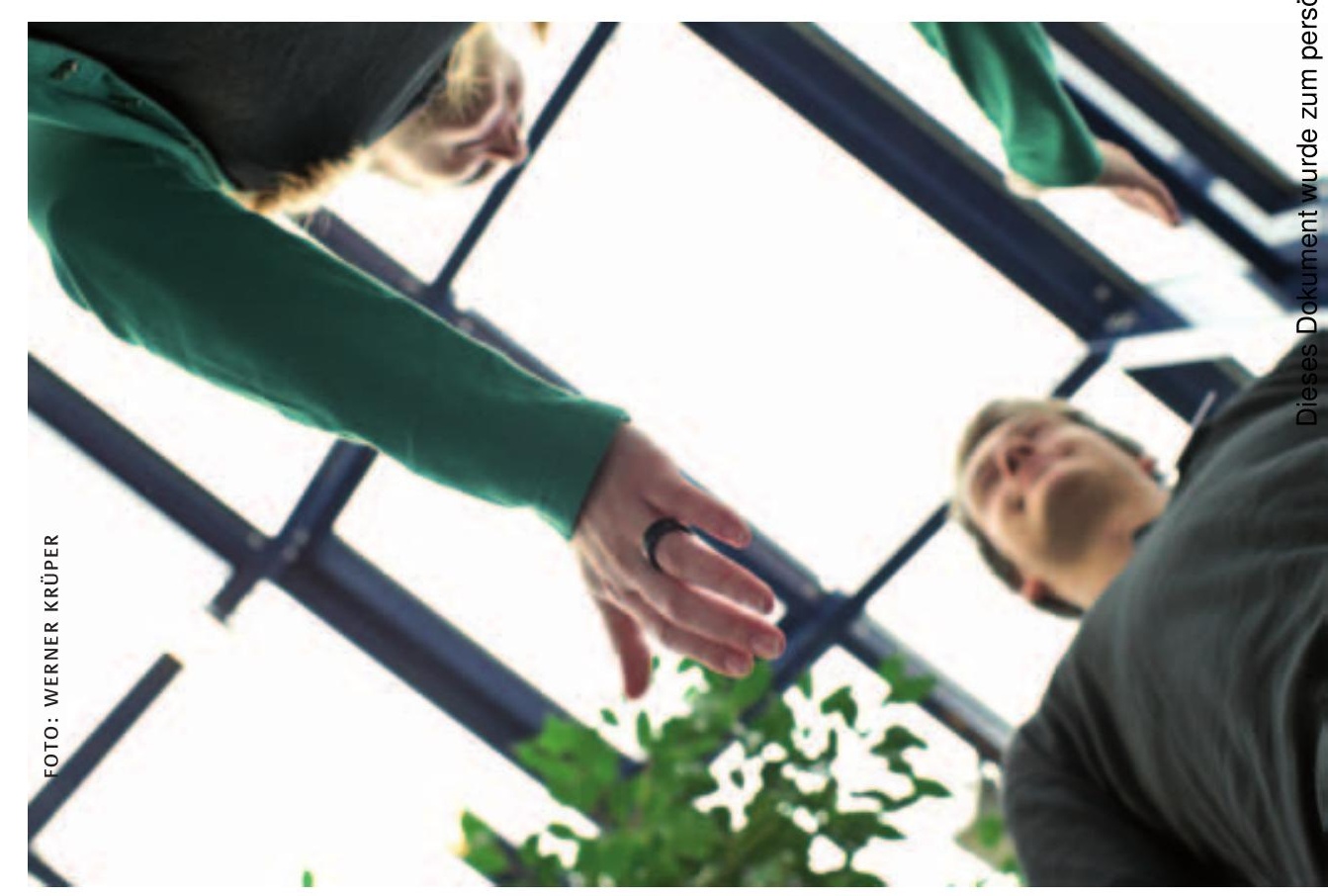
wie folgt zusammen: „[...] indem die Beteiligten an einem Gespräch auf eine ganz bestimmte Weise miteinander reden, stellen sie den Rahmen (Goffman) ihres Gespräches jeweils selbst her, legen fest, ob es sich um einen informellen small talk oder etwa um ein Prüfungsgespräch handelt.“ Beheimatet ist der Rahmenbegriff in der Soziologie, die nach dem kollektiven Wissensbestand einer Gesellschaft fragt.

Einen anderen Zugang zur Kontextualisierung wählt dagegen Peter Auer [1, 2], 
wenn er nicht von Rahmen, sondern von "Schemata“ spricht. Der Schemabegriff wurde bereits 1932 von F.C. Bartlett [3] etabliert und hat später in der kognitiven Psychologie eine große Bedeutung erlangt. Wie die „Rahmen“ in der Soziologie, können auch Schemata als Erwartungsmuster bezeichnet werden - nur werden Schemata von der Psychologie als Bestandteile der individuellen Kognition eines Menschen betrachtet. Sie sind theoretisch modellierbar und der experimentellen Forschung zugänglich.

Peter Auer nimmt nun jedoch einen Brückenschlag von der kognitiven Psychologie zur interaktionalen Soziologie vor, indem er feststellt: „Schemata sind nicht nur eine kognitive, sondern auch eine interaktive Realität. Dies bedeutet, dass sich alle Teilnehmer auf die Gültigkeit eines bestimmten Schemas einigen müssen.“ [1, S. 25f.] Konkret nimmt Auer Schemata und die damit verbundenen interaktiven Problemstellungen aus fünf Kommunikationsbereichen in den Blick. Diese fünf Bereiche möchten wir im Folgenden näher betrachten, weil sie sich in besonderer Weise dazu eignen, die Kommunikationspraxis in der stationären Akutpsychiatrie zu hinterfragen.

\section{Wie funktioniert Kommunikation? Fünf Schemata}

Erstens: Damit ein Gespräch gelingen kann, muss eine auf den ersten Blick banal erscheinende Voraussetzung erfüllt sein: Die Gesprächspartner müssen sich bewusst darüber sein, dass sie miteinander kommunizieren. In Anlehnung an Goffman [10] spricht Auer in diesem Zusammenhang vom Schema des fokussierten Interagierens. Verbunden damit ist die Frage: Reden wir (gerade) miteinander? Zum gesellschaftlichen Wissensbestand gehören bestimmte Kontextualisierungshinweise, die erkennen lassen, dass ein Gespräch beabsichtigt ist, aktuell abläuft oder beendet werden soll. Konkret beinhalten diese zum einen sog. Vorlaufaktivitäten wie (in der Face-to-faceKommunikation) das Aufnehmen von Blickkontakt. Initiiert wird das Gespräch sodann durch Grußsequenz und persönliche Anrede, also beispielsweise: „Guten Morgen, Frau Müller!“ Während des Gespräches belegt die fortwährende gegenseitige Bezug-

nahme auf das vom Gegenüber Gesagte (im linguistischen Terminus: Kohärenzstiftung), dass ein gemeinsames, reziprokes Handeln geschieht. Vor dem Abschluss finden oft Beendigungsvorlaufstrukturen Verwendung, etwa die Bemerkung: „So, dann haben wir für den Moment alles Wichtige geklärt.“ Die finale Klammerung der Interaktion erfolgt durch eine abschließende Grußsequenz und die Beendigung des Kontaktes - in der Face-to-faceKommunikation wenden sich die Gesprächspartner in der Regel voneinander ab. Was hier so selbstverständlich klingt, kann in

Was aus der Sicht der Behandler als unkooperatives Verhalten erscheint, ist aus der Perspektive des Patienten lediglich die Ablehnung des von außen vorgegebenen Handlungsschemas. der Praxis einige Stolpersteine beinhalten. Auf entsprechende Erfahrungen in und mit der Psychiatrie werden wir unten im Rahmen der „Beobachtungen“ näher eingehen.

Zweitens: Auch wenn die gegenseitige Aufmerksamkeit und das gemeinsame Ziel, aufeinander Bezug nehmend zu kommunizieren, gegeben sind, bedarf die Interaktion bestimmter Organisationsprinzipien. Dazu gehört insbesondere das Schema des Turn taking, verbunden mit der Frage: Wer spricht (gerade) mit wem? Schon in einem Zweier-Gespräch (und noch viel mehr in größeren Runden) muss es Regeln geben, die sicherstellen, dass nicht alle Beteiligten gleichzeitig das Wort ergreifen. Wer aktuell die Rolle des Sprechers und wer die des Hörers einnimmt, wird deshalb durch bestimmte Signale (Kontextualisierungshinweise) bestimmt. So signalisiert der Hörer beispielsweise seine Aufmerksamkeit durch „Backchannels“ oder „Continuers“ wie „Mhm“, „Ja“, „Ach!“ etc. Er kann aber auch selbst einen Gesprächsbeitrag („Turn“) beginnen und damit die Rolle des Sprechers beanspruchen. Wichtig ist dabei, dass grundsätzlich alle an der Interaktion Beteiligten das gleiche Recht und die gleichen Möglichkeiten haben, als Sprecher bzw. als Hörer zu fungieren. Allerdings gibt es soziale Kontexte, in denen diese Gleichberechtigung nicht oder zumindest nur eingeschränkt gilt. So schreibt Uta Quasthoff dem Arzt in Visitengesprächen das Recht des „primären Sprechens“ zu [17, S. 77].
Auch diesen Punkt werden wir mit Blick auf die Psychiatrie noch näher betrachten.

Drittens: Die Erwartungen an den Ablauf der Kommunikation werden wesentlich durch Handlungsschemata geprägt, also durch die Frage: Was tun wir (gerade) miteinander? Peter Auer unterscheidet hier zwischen komplexen Handlungstypen (Gattungen) und einfachen Handlungstypen, die sich wechselseitig beeinflussen: „Gattungen bestimmen die einfachen Handlungstypen mit, die in ihnen und als ihr Bestandteil ablaufen, während umge-

kehrt die in einer Gattung möglichen oder notwendigen Handlungen mit zur Definition der Gattung gehören“ [1, S. 34]. Bezogen auf die Psychiatrie bedeutet das beispielsweise, dass zum komplexen Handlungstyp der Anamnese einfache Handlungstypen wie das Beantworten von Fragen zur Person, zur Krankheitsgeschichte etc. sowie in der Regel auch das Ausfüllen von Fragebögen gehören. Eingeleitet, also kontextualisiert, werden diese Schritte normalerweise durch explizite Ankündigungen der Mitarbeitenden: „Wir brauchen noch einige Angaben von Ihnen!" Gerade wenn ein Patient unfreiwillig in psychiatrische Behandlung geraten ist, kann es sein, dass er sich weigert, die gewünschten Auskünfte zu erteilen. Was aus der Sicht der Behandler als unkooperatives Verhalten erscheint, ist aus der Perspektive des Patienten dann lediglich die Ablehnung des von außen vorgegebenen Handlungsschemas.

Viertens: Die Frage „Worüber reden wir (gerade) miteinander?" betrifft die thematischen Schemata. Hierzu stellt Auer [1, S. 39] fest: „Inhaltlich gesehen lassen sich Themen im Sinne der Topik als Schemata verstehen, die den Gesprächsteilnehmern bestimmte ,Gemeinplätze‘ zur Verfügung stellen." So wird ein verhaltenstherapeutischer Diskurs über das Thema „Angst“ mit hoher Wahrscheinlichkeit Begriffe wie „Vermeidungsverhalten“ und „Verstärkung“ enthalten. Umgekehrt zeigt in der Frage „Was tippen Sie denn für heute 
Abend?" das Verbum, dass es wohl nicht um ein medizinisches Thema geht. Dass thematische Schemata in der Psychiatrie nicht immer frei aushandelbar sind, sondern vom Therapeuten gesteuert werden, zeigt eine empirische Untersuchung von Rüdiger Weingarten zu Reformulierungen in der Gesprächspsychotherapie. Demnach „geht es in einer Einzeltherapie darum, dass der Klient seine Probleme bearbeitet. [...] Redet der Klient daher über das Handeln anderer und nicht über sein Handeln oder zumindest über sein Erleben der Handlungen anderer, so fokussiert der Therapeut wiederum ihn selbst.“ [23, S. 239]

Fünftens: Der letzte von Peter Auer genannte Bereich betrifft die Beziehungsschemata und die Frage: Wie stehen wir (gerade) zueinander? Hier besteht ein enger Bezug zum Beziehungsaspekt einer Botschaft, wie ihn Paul Watzlawick [22] und Friedemann Schulz von Thun [19] beschrieben haben. Allerdings geht es dort nur um die Wirkungsrichtung vom Sender zum Adressaten; die Botschaft vermittelt also Informationen darüber, wie der Sprecher seine Beziehung zum Gegenüber sieht. Innerhalb des Kontextualisierungsansatzes liegt der Schwerpunkt hingegen auf der Frage, welche Beziehung der Interaktanten durch die Kommunikation entsteht. Die Dynamik des Geschehens findet somit stärkere Beachtung. Armin Körfer et al. wenden diese Perspektive auf Formen der Arzt-Patienten-Beziehung an, wenn sie feststellen: „Im Gegensatz zum paternalistischen Modell [...] beruht das Partnerschaftsmodell auf diskursiver Verständigung zwischen gleichberechtigten Partnern. [...] Für dieses Modell der Arzt-Patienten-Kommunikation ist seine Diskursivität insofern konstitutiv, als sowohl Arzt als auch Patient vorgängige Überzeugungen davon, was denn zum Besten des Patienten sei, zu revidieren bereit sind.“ $[14$, S. 64f.]

Einen Überblick über die fünf genannten Schemata bietet Tab. 1.

\section{Wie kommen Sie bloß darauf? Zur Methode der Teilnehmenden Beobachtung}

Wie lassen sich diese theoretischen Grundlagen nun für die Analyse der Kommunikationspraxis in der Akutpsychiatrie nutzbar

Tab. 1 Die fünf Kontextualisierungsschemata nach Peter Auer im Überblick

\begin{tabular}{|lll}
$\begin{array}{l}\text { Kontextualisierungs- } \\
\text { schema }\end{array}$ & Frage & Betrifft z. B. \\
\hline $\begin{array}{l}\text { Schema des fokus- } \\
\text { sierten Interagierens }\end{array}$ & $\begin{array}{l}\text { Reden wir (gerade) } \\
\text { miteinander? }\end{array}$ & $\begin{array}{l}\text { Grußsequenzen, gegenseitige Bezug- } \\
\text { nahme, Aufmerksamkeitssteuerung }\end{array}$ \\
\hline $\begin{array}{l}\text { Schema des Turn } \\
\text { taking }\end{array}$ & $\begin{array}{l}\text { Wer spricht (gerade) } \\
\text { mit wem? }\end{array}$ & $\begin{array}{l}\text { Etablierung von Sprecher- und Hörer- } \\
\text { rolle, Strategien des Rollenwechsels }\end{array}$ \\
\hline Handlungsschemata & $\begin{array}{l}\text { Was tun wir (gerade) } \\
\text { miteinander? }\end{array}$ & $\begin{array}{l}\text { Einfache Handlungstypen (z. B. Klä- } \\
\text { rung offener Fragen), komplexe Hand- } \\
\text { lungstypen (z. B. Psychoedukatives } \\
\text { Seminar) }\end{array}$ \\
\hline $\begin{array}{l}\text { Thematische Sche- } \\
\text { mata }\end{array}$ & $\begin{array}{l}\text { Worüber reden wir } \\
\text { (gerade) miteinan- } \\
\text { der? }\end{array}$ & $\begin{array}{l}\text { Themenbereiche, mit denen be- } \\
\text { stimmte Phrasen/Formulierungsmus- } \\
\text { ter verbunden sind }\end{array}$ \\
\hline Beziehungsschemata & $\begin{array}{l}\text { Wie stehen wir (gera- } \\
\text { de) zueinander? }\end{array}$ & $\begin{array}{l}\text { Explizite und implizite Botschaften } \\
\text { zum Verhältnis der Kommunikations- } \\
\text { partner }\end{array}$ \\
\hline
\end{tabular}

machen? Unser Vorgehen basiert im Prinzip auf der Methode der „Teilnehmenden unstrukturierten Beobachtung“ (TUB), die der Kulturanthropologie und der sozialwissenschaftlichen Feldforschung entstammt. Grundlegend ist die Annahme, dass erst die unmittelbare Beteiligung an einer Situation es ermöglicht, die Vielzahl relevanter Aspekte in ihrer Gesamtheit und in ihrem Aufeinander-Bezogensein wahrzunehmen. Deshalb verzichtet die TUB bewusst auf die Vorgabe von Beobachtungsmethoden und untersuchungsleitenden Hypothesen.

Die Praxis der TUB beschreibt Roland Girtler wie folgt: „Der qualitativ Forschende ist $[\ldots]$ in dem betreffenden sozialen Feld selbst aktiv. Er knüpft Kontakte, spricht mit Leuten, lässt sich in die verschiedenen Rituale des Handelns einweihen und interpretiert schließlich aufgrund seiner Beziehung zum sozialen Ganzen seine Beobachtungen und Interviews“ [8, S. 110]. Um die Situation nicht zu verfälschen, fertigt der Forscher keine Tonbandoder Videoaufnahmen an, sondern notiert höchstens Stichwörter, aus denen er dann später (Gedächtnis-)Protokolle erstellt. Dieses Vorgehen hat den Vorteil, der Lebenswirklichkeit in deutlich höherem Maße gerecht zu werden, als es Experimente und kontrollierte Studien vermögen.

Wir haben nun in unserer Arbeit diesen Gedankengang noch einen Schritt weiter geführt und das Vorgehen innerhalb der TUB nochmals variiert. In der Regel sieht die TUB vor, dass der Forscher sich von außen in sein Untersuchungsfeld begibt. Das hat zum einen den Vorteil, dass er durch seine wissenschaftliche Legitimation leichter Zugang zu einer Vielzahl von Informationsquellen hat, im klinischen Bereich also beispielsweise auch an Mitarbeiterbesprechungen teilnehmen und ggf. sogar Akteneinsicht erhalten kann. Darüber hinaus erleichtert es die Rolle als eigentlich Außenstehender, unvoreingenommen an das Geschehen heranzugehen und das Erlebte immer wieder mit einer gewissen inneren Distanz zu reflektieren. Mit Blick auf die Untersuchung von Kommunikation in der Akutpsychiatrie besteht der Nachteil der klassischen TUB jedoch darin, dass nur zwei Perspektiven Berücksichtigung finden können: entweder die des neutralen Beobachters, der sich ein Bild von der Gesamtsituation macht, oder die des Mitarbeiters - Letzteres ist dann der Fall, wenn der Forscher, eine entsprechende Ausbildung vorausgesetzt, während der Beobachtung in der Rolle eines Krankenpflegers agiert. Das Erleben des Patienten hingegen wird allenfalls in Gesprächen mit dem Forscher ermittelt, wodurch notwendig eine Fragmentierung und ein erheblicher Informationsverlust entstehen.

Was wäre aber die Alternative? Wollte eine reale Patientin zugleich als Forscherin agieren, würde sie zwangsläufig mit dem Vorwurf konfrontiert, sie sei krankheitsbedingt gar nicht zur Reflexion in der Lage 
und bringe zudem nicht die erforderliche innere Distanz mit. Allerdings ist es ein Charakteristikum jeder TUB, dass Objektivität immer nur eingeschränkt erreichbar ist, denn durch das Miterleben einer Situation entsteht notwendig ein subjektiv gefärbter Eindruck. Und aus der Tatsache, dass eine akute Krisensituation das subjektive Erleben von Kommunikation in besonderer Weise prägt, lässt sich nicht folgern, dass dieses Erleben defizitär oder belanglos wäre.

Vor dem Hintergrund dieser Erwägungen möchten wir im Folgenden einige Erfahrungen in den Blick nehmen, welche die Erstautorin dieses Aufsatzes während eines mehrmonatigen Aufenthaltes als Patientin auf einer akutpsychiatrischen Depressions- und Krisenstation gemacht habe. Technisch gesehen handelt es sich dabei um die Post-hoc-Auswertung einer Quasi-TUB aus Patientenperspektive. Durch die eigene Betroffenheit ist die Darstellung naturgemäß stärker personengebunden, als dies bei einer klassischen TUB der Fall wäre. Wir sind uns dieser Tatsache bewusst und schreiben unserem Vorgehen deshalb auch nicht den Status wissenschaftlicher Empirie im strengen Sinne zu. Dennoch sind wir der Meinung, dass gerade in der Psychiatrieforschung die Teilnehmende Beobachtung aus Betroffenenperspektive eine gewinnbringende Methode in Ergänzung des bewährten Methodenkanons sein kann. Denn nur auf diese Weise lässt sich ein unverfälschter Eindruck von dem gewinnen, worum es doch letztlich der Psychiatrie im Kern gehen sollte: um das reale Erleben von Patientinnen und Patienten.

Und was ist dabei herausgekommen? Beobachtungen aus der Akutpsychiatrie

Weil naturgemäß keine unmittelbaren Aufzeichnungen oder Tonbandmitschnitte vorgenommen werden konnten (und dies, wie oben erläutert, auch nicht dem Vorgehen einer TUB entspräche), lassen sich im Folgenden nur Eindrücke aus der Makroperspektive schildern. Allerdings kommt diese Gesamtschau der tatsächlichen menschlichen Wahrnehmung wohl auch näher als ein Herausschneiden einzelner Aspekte der Kommunikation. Inhaltlich orientieren wir uns an den oben genannten Kontextualisierungs-Schemata nach Peter Auer (vgl. die obige Tab. 1). Vor deren Hintergrund soll jeweils gefragt werden, welche Art von Situation durch die Kommunikation geschaffen wird.

\section{Beobachtungen zum fokussierten Interagieren}

Beginnen wir also mit dem Schema des fokussierten Interagierens und der Frage: Reden wir gerade miteinander? Hier fällt auf, dass das Initiierungsrecht für Gespräche grundsätzlich bei den Mitarbeitern liegt: Sie können und dürfen jeden Patienten jederzeit ansprechen. Im Beobachtungszeitraum zeigte sich jedoch, dass dieses Recht in den allermeisten Fällen behutsam wahrgenommen wurde: Vor dem Betreten
Ein Abblocken erfolgte meist dadurch, dass die Mitarbeiter verbal oder non-verbal ignalisierten, gerade anderes zu tun zu haben. war noch eine andere Frage. Der Zugang zu den Therapeuten war noch schwieriger, da für jeden Patienten etwa eine halbe Stunde Gesprächszeit pro Woche (sowie zehn Minuten Oberarztvisite) vorgesehen war. Insofern machten diejenigen Patienten, die sich nicht ohnehin resigniert zurückzogen, immer wieder die Erfahrung, dass ihre Versuche zur Anbahnung einer fokussierten Interaktion abgeblockt wurden.

Ein solches Abblocken erfolgte meist dadurch, dass die Mitarbeiter verbal oder non-verbal signalisierten, gerade anderes zu tun zu haben. Die non-verbale Komponente umfasste dabei beispielsweise körperliches Abwenden und die Beschäftigung mit anderen Tätigkeiten noch während des Sprechens. Hinzu kamen gerade bei besonders engagierten Mitarbeitern auch bedauernde Gesten und paraverbale Signale wie Seufzen. Wesentlich brüsker war eine andere Art der Abweisung: Es gab einige Mitarbeiter, die sich auch während ruhigerer Phasen ihres Dienstes, also beispielsweise am Wochenende, grundsätzlich hinter die von Patientenzimmern wurde angeklopft und auf Terminvorschläge, auch für Therapiegespräche, folgte in der Regel die Frage „Passt Ihnen dieser Termin?“ Dieses Vorgehen lässt sich als (durchaus erfolgreicher) Versuch interpretieren, die im Prinzip gegebene Hierarchie abzuschwächen und den Patienten als Gesprächspartner ernst zu nehmen. Konterkariert wurde dieses Bestreben allerdings dadurch, dass es für die Patienten oft schwierig war, ihrerseits Gesprächskontakte zu initiieren. Ein Grund dafür lag in der hohen Arbeitsbelastung der Mitarbeiter. Bei einer Relation von in der Regel zwei diensthabenden Pflegemitarbeitern und 24 Patienten - unter denen zudem eine hohe Fluktuation herrschte - waren intensive persönliche Gespräche kaum möglich. Der Versuch, dies durch ein Bezugspflegesystem zu kompensieren, krankte daran, dass fast alle Mitarbeiter nur in Teilzeit beschäftigt, also nicht regelmäßig vor Ort waren. Wenn ein Patient gerne mit seiner Bezugspflegerin sprechen wollte, musste er oft mehrere Tage warten, bis diese überhaupt wieder im Dienst war - ob sie dann auch Zeit hatte, geschlossene Tür des Stationszimmers zurückzogen. Diese physische Barriere hielt die meisten Patienten von vornherein von Versuchen zur Kontaktaufnahme ab. (Andere Mitarbeiter dagegen nutzten Freiräume konsequent dazu, um gerade auch mit zurückhaltenden Patienten ins Gespräch zu kommen. Wo dies nicht unmittelbar verbal gelang, dienten manchmal auch gemeinsame Spiele der Kommunikationsanbahnung.)

Nicht selten ergab sich der Eindruck, dass von Patienten initiierte Kontakte eher als Störung des Stationsablaufs denn als berechtigtes Anliegen gewertet wurden. Das stand in krassem Gegensatz zu der Aufforderung, sich in Krisensituationen doch unbedingt rechtzeitig an die Mitarbeiter zu wenden. Die Patienten standen damit vor einem Dilemma: Meldeten sie sich bei Problemen nicht, so galt das als Symptom eingeschränkter Kommunikationsfähigkeit. Suchten sie dagegen das Gespräch, so liefen sie Gefahr, ständig abgewiesen zu werden, was den Stresslevel weiter erhöhte. Manche Patienten versuchten in ihrer Not schließlich, massiv auf 
gen blieben. So war es üblich, dass ein neu aufgenommener Patient von einer Mitarbeiterin über die Station geführt und in den formalen Tagesablauf, also etwa die Essenszeiten und den Termin der obligatorischen Morgenrunde, eingewiesen wurde. In Therapiegruppen begrüßte die Gruppenleiterin neue Teilnehmer und gab einen kurzen Überblick über die Arbeitsweise und die Regeln der Gruppe. Diverse untergeordnete Handlungsschritte wurden vom Stationsablauf bestimmt (z.B. die durch Aushang festgelegten Zeiten der Medikamentenausgabe). Hinweise zu anstehenden Aktivitäten gaben die Mitarbeiter in Aussage- oder Anweisungsform, z.B. mit der Feststellung: „Sie haben jetzt Ergotherapie! “ Patienten dagegen nahmen auf Handlungsmuster meist in Frage- oder Bittform Bezug, also mit Formulierungen wie „Könnte ich wohl ...?“, „Soll ich ...?" oder „Darf ich ...?”.

Generell erhielten die

Patienten anscheinend genau die Informationen, die erforderlich waren, um sie möglichst nahtlos in die Klinikroutine einzupassen. Offen blieb jedoch, was diese Routine überhaupt mit dem einzelnen Patienten zu tun hatte und inwiefern er davon individuell profitieren sollte. Das übergeordnete (Be-)Handlungsschema der Station sah offenbar zwei getrennte Informationsflüsse vor: Zum einen gab es feste Vorgaben darüber, welchen therapeutischen oder sonstigen Maßnahmen sich ein Patient zu unterziehen hatte, und diese Vorgaben wurden über die Mitarbeiter zu den Patienten kommuniziert. In Gegenrichtung wurden vom Patienten zahllose persönliche Informationen gesammelt, deren weitere Verwendung - und deren Bezug zur Therapie - jedoch weitgehend im Dunkeln blieben. Deshalb herrschte unter vielen Patienten eine permanente Verunsicherung darüber, wie es denn nun eigentlich mit ihnen weitergehen sollte.

Besonders angstbesetzt waren die wöchentlichen Oberarzt-Visiten, in denen über den Entlassungstermin entschieden wurde: Wer nämlich im Rahmen einer Krisenintervention (freiwillig) aufgenommen worden war, sollte möglichst nicht zu lange in der Klinik bleiben, um einen Hos- pitalisierungseffekt zu vermeiden. Dieser eigentlich sehr sinnvolle Grundsatz führte jedoch auf einer Station, die unter chronischem Personalmangel in Pflege und Therapie litt, letztlich dazu, dass Patienten nur ein paar Tage oder höchstens ein paar Wochen „verwahrt“ (gleichlautendes Zitat mehrerer Patienten und Mitarbeiter) wurden, in dieser Zeit in einem Standardprogramm mitliefen und anschließend wieder in ihr - unverändert problembeladenes - Leben entlassen wurden. Eine weitere Begleitung durch aufsuchende Pflege war nicht vorgesehen. Dieses Grundmuster führte auch bei vielen engagierten Pflegemitarbeitern zu einem hohen Frustrationsgrad, weil sie ihr Ziel, Menschen zu helfen, kaum erreichen konnten.

arbeitern und Patienten naturgemäß sehr häufig um den Themenkomplex „Krankheit und Krankheitsbewältigung“. Dabei fiel auf, dass die Kommunikation in weiten Teilen hierarchisch strukturiert war: Welche Aspekte relevant waren und wie man diese zu beurteilen hatte, gaben die Mitarbeiter vor. Zur Informationsvermittlung gab es unterschiedlich ausgerichtete Gruppen. In der Depressionsgruppe hatten die Patienten einmal wöchentlich die Möglichkeit, eineinhalb Stunden lang über ein (krankheitsbezogenes) Thema ihrer Wahl zu sprechen. $\mathrm{Zu}$ den häufigsten Themen, die sich im Abstand einiger Wochen regelmäßig wiederholten, gehörten beispielsweise der Umgang mit Antidepressiva, der langfristige Verlauf von Depressionen oder die Frage, inwieweit es sinnvoll sei, im persönlichen Umfeld über die eigene Erkrankung zu sprechen. Die Gruppenleitung wechselte gelegentlich, wurde aber meist von einem Diplom-

Diese allgemeinen Beobachtungen bedürfen allerdings punktuell einer Differenzierung. So war immer wieder das Bemühen erkennbar, Patienten individuell gerecht zu werden - beispielsweise durch spezielle Angebote wie Einzel-Musiktherapie und Einzel-Bewegungstherapie. Auch fanden Ärzte, Therapeuten, Pflegemitarbeiter und Patienten manchmal gemeinsam kreative und individuelle Lösungen, um nach einem langen Klinikaufenthalt den Übergang in den Alltag zu erleichtern. Verunsichernd für die Patienten war jedoch, dass diese Abläufe keiner erkennbaren Logik unterlagen, dass sich also ein generelles, übergreifendes Handlungsschema nicht identifizieren ließ. Entsprechend entstand oftmals der Eindruck, dass diejenigen Patienten im Vorteil waren, die für ihre Bedürfnisse und Anliegen eintreten konnten. Wer dazu (auch krankheitsbedingt) nicht in der Lage war, durchlief mehr oder weniger lautlos eine Maschinerie, die ihn schließlich unverändert wieder ausspuckte.

\section{Beobachtungen}

zu den thematischen Schemata

Neben der Klärung organisatorischer Fragen drehten sich Gespräche zwischen Mit-
Psychologen übernommen, der jede Sitzung mit der Bemerkung einleitete: „Jetzt haben Sie die Möglichkeit, von einem Experten fundierte Informationen zu erhalten. Nutzen Sie sie!“ Der Experte war er selbst. Entsprechend dienten die Runden dann auch weniger dem Erfahrungsaustausch als der Erarbeitung von Erkenntnissen, die an einer Tafel festgehalten wurden.

Noch stärker schulisch angelegt war die psychoedukative Gruppe, in der an jedem Mittwochnachmittag über das Wesen von Depressionen unter besonderer Berücksichtigung des Zusammenhangs von Denken, Handeln und Fühlen informiert wurde. Hier gab es ein festes Curriculum und schriftliches Unterrichtsmaterial. Ein Bezug zwischen dem vermittelten Wissen und der individuellen Situation der Patienten war jedoch kaum erkennbar. So beschwerten sich Patienten in Gesprächen untereinander immer wieder darüber, dass sie sich durch die Belehrungen zum „Depressionsdreieck“ oder zur depressiven „Abwärtsspirale“ unverstanden, ja geradezu verhöhnt fühlten. Gerade diejenigen, die angesichts persönlicher Schicksalsschläge in eine Krise geraten waren, fanden sich und ihr Erleben in den vermittelten Inhalten überhaupt nicht wieder. 
Die starke Tendenz zur Deindividualisierung war auch in einem nicht geringen Teil der Einzelgespräche mit Therapeuten und Pflegemitarbeitern erkennbar. Vermutlich aus Gründen der Zeitökonomie wurden Versuche, die Erkrankung im Rahmen der eigenen Lebensgeschichte zu betrachten, meist konsequent abgeblockt. Als relevant galten nur bestimmte, von den Mitarbeitern vorgegebene Daten und Fakten, die entsprechend schematisch erhoben wurden. Die Patienten erhielten deshalb regelmäßig diverse Formblätter, die nach festen Vorgaben auszufüllen waren. Abgefragt wurden beispielsweise die aktuelle Stimmung (auf einer Skala von 1-10) oder Gedanken und Handlungen, die der jeweils aktuellen Gefühlslage vorausgingen (dass zu komplexe Gedankengänge dabei unerwünscht waren, machte die extrem schmale Tabellenspalte mehr als deutlich). Tiefer-

gehende Fragen wie die nach dem individuellen Lebenssinn des Patienten, seiner Lebenserfahrung,

\section{Manche Mitarbeiter lernten den} Patienten als Menschen kennen, nicht nur als Symptomträger.

\section{Beobachtungen} zu den Beziehungsschemata

In der Psychiatrie stellen die Beziehungsschemata den zentralen Aspekt der Kommunikation zwischen Mitarbeitern und Patienten dar. Gerade hier spielen neben den expliziten Botschaften - also dem direkt Gesagten - auch die impliziten Botschaften eine große Rolle. Letztere umfassen all das, was sich aus dem gesamten Verhalten einer Person gegenüber einer anderen ergibt. Wer sich in stationärer Behandlung befindet, ist gegenüber solchen Botschaften in der Regel besonders empfindsam, denn seinen Werten, Normen und Wünschen tauchten dagegen nicht auf.

Auch hier ist jedoch ausdrücklich anzumerken, dass es unter den Therapeuten und Pflegemitarbeitern einige Gegenden-Strom-Schwimmer gab, die sich sehr bemühten, jeden Patienten als Individuum wahrzunehmen. So kam es im Beobachtungszeitraum immer wieder auch zu persönlichen Gesprächen auf Augenhöhe, die von ehrlichem gegenseitigen Interesse geprägt waren. Die Themen gingen dabei teilweise weit über Krankheit und Behandlungsstrategien hinaus und betrafen auch ganz konkrete Lebenserfahrungen. Diese persönliche Kommunikation erwies sich in mehrfacher Hinsicht als therapeutisch nützlich: Zum einen lernten die betreffenden Mitarbeiter den Patienten als Menschen kennen, nicht nur als Symptomträger. Das auf diesem Wege erworbene Wissen dürfte in manchen späteren Situationen das Verständnis erleichtert und individuelle therapeutische Maßnahmen ermöglicht haben. Zum anderen erhielt der Patient gerade im persönlichen Austausch ein Ausmaß an Lebenshilfe, das er „verfügt über eine erhöhte Wahrnehmungssensibilität, die sich auf den Interaktionspartner richtet“ [7, S. 92]. Deshalb gilt im Folgenden dem implizit Vermittelten besondere Aufmerksamkeit.

Entsprechend der von Friedemann Schulz von Thun [19] vorgenommenen Unterscheidung von „Du-Botschaften“ und „Wir-Botschaften“ soll zunächst gefragt werden, wodurch zum Ausdruck kommt, was der Sender vom Empfänger hält. Für den Beobachtungszeitraum ist festzustellen, dass die expliziten Aussagen von Mitarbeitern gegenüber Patienten oft betont wertschätzend waren. Die Wirkung solcher Botschaften wurde jedoch nicht selten durch den erkennbar therapeutischen Duktus gemindert: Wer regelmäßig schon beim geringsten Anlass (oder Nicht-Rückschritt) die Standardaussage „Das haben Sie gut gemacht!“ zu hören bekam - professionell begleitet durch Blickkontakt, Lächeln und ein aufmunterndes Nicken -, der fühlte sich eher wie ein entwicklungsgestörtes Kind behandelt statt wie ein mündiger, erwachsener Mensch. Dabei machte es einen deutlichen Unterschied, ob der- artige positive Rückmeldungen von Mitarbeitern kamen, zu denen kaum persönlicher Kontakt bestand, oder von solchen, die sich für den Patienten als Menschen interessierten. Letztere waren in ihren Aussagen wesentlich glaubwürdiger, zumal sie auf Standardformulierungen verzichten und ihre Anerkennung und Wertschätzung ganz individuell zum Ausdruck bringen konnten.

Während also die Mitarbeiter erkennbar bemüht waren, explizit positive, aufmunternde und selbstwertstärkende DuBotschaften zu vermitteln, beinhalteten die impliziten Botschaften nicht selten das genaue Gegenteil. Zu diesem Eindruck trug schon die verwendete Fachsprache bei. So hieß die für einen Patienten zuständige Ärztin oder Therapeutin „Fallverantwortliche“, womit gleich zwei Punkte geklärt waren: Erstens, der Patient wurde nicht als Mensch, sondern als Fall gesehen, und zweitens, die Verantwortung für alles Geschehen lag ausschließlich bei der professionellen Kraft.

Ein weiterer aussagekräftiger Begriff bezog sich auf die unerfreuliche Situation, dass es einer Patientin gerade psychisch ausgesprochen schlecht ging. Wer beispielsweise heftig weinte und kurzfristig nicht zu einem Gespräch in der Lage war, der musste sich anschließend mit der Frage auseinandersetzen, warum er denn nun „dekompensiert" habe. Diese Formulierung ist nicht nur emotional kalt, sie vermittelt durch die negative Vorsilbe „de-“ auch den Eindruck, die Patientin habe sich eines Versagens schuldig gemacht. Auch hier ist festzustellen, dass es sich nicht nur um das subjektive Empfinden einer einzelnen Person handelt. Vielmehr stellt Hilde Schädle-Deininger mit Blick auf den Sprachgebrauch in der psychiatrischen Pflege fest: „Durch eine versachlichte Sprache wird der Mensch eher zum Objekt. Der Mensch wird auf seine Funktionsfähigkeit und sein Angepasstsein reduziert, es kommen weniger die annehmenden, fürsorglichen und kommunikativen Bedürfnisse zum Tragen, die Sprache wird weitgehend funktionalisiert.“ [18, S. 182]

Die implizite Botschaft, dass Patienten sich durch ihre Erkrankung eines Fehlverhaltens schuldig machten, wurde 
im Übrigen permanent durch die verhaltenstherapeutische Konzeption gestützt. Der lerntheoretische Ansatz besagte, dass erwünschtes Patientenverhalten durch Belohnungen verstärkt, unerwünschtes, „dysfunktionales“ Verhalten hingegen durch Bestrafung eliminiert werden sollte. Die Umsetzung erfolgte grundsätzlich arbeitsteilig: Für Belohnungen waren die Patienten selbst zuständig („Tun Sie sich etwas Gutes!“ oder „Belohnen Sie sich!“). Verhängung und Vollzug von Strafen oblagen dagegen den Behandlern (wörtliches Zitat des Oberarztes gegenüber einer Patientin: „Ich habe mir überlegt, was Ihnen wohl am meisten weh tut.“).

Interessanterweise bestritten einige Ärzte und Therapeuten vehement, dass in ihrer Klinik mit Bestrafungen gearbeitet werde. Das ist in der Sache eindeutig falsch, weil das Modell von Burrhus F. Skinner [20, 21], das den lerntheoretischen Therapieansätzen zugrundeliegt, zu den Methoden des operanten Konditionierens ausdrücklich auch die Bestrafung zählt. Guy R. Lefrancois [15, S. 117] fasst dies wie folgt zusammen: „Verstärker können positiv sein (wirksam durch ihre Darbietung; Belohnung) oder negativ (wirksam durch ihre Wegnahme; Entlastung). Bestrafung II oder Entzugsbestrafung bedeutet, auf das Verhalten hin eine erfreuliche Konsequenz zu entfernen; Bestrafung I oder Präsentationsbestrafung bedeutet, eine aversive Konsequenz zu präsentieren.“ In diesem Sinne wurden im Beobachtungszeitraum wiederholt gezielt Bestrafungen als therapeutische Maßnahmen ein-

gesetzt, was mehrere Patienten in verschiedenen Situationen und unabhängig voneinander als „demütigend“ bezeichneten. Zugleich entzogen sich die Mitarbeiter aber jeder Diskussion über ihr Vorgehen, indem sie entweder behaupteten, es gebe in der Verhaltenstherapie keine Bestrafungen, oder schlicht feststellten: „Ich habe keine Lust, mit Ihnen über Therapiekonzepte zu diskutieren!“

Völlig andere Du-Botschaften wurden in einzelnen Begleitangeboten vermittelt, insbesondere in der Musiktherapie, der Bewegungstherapie und der Selbstbehauptungsgruppe für Frauen. Diese Gruppen

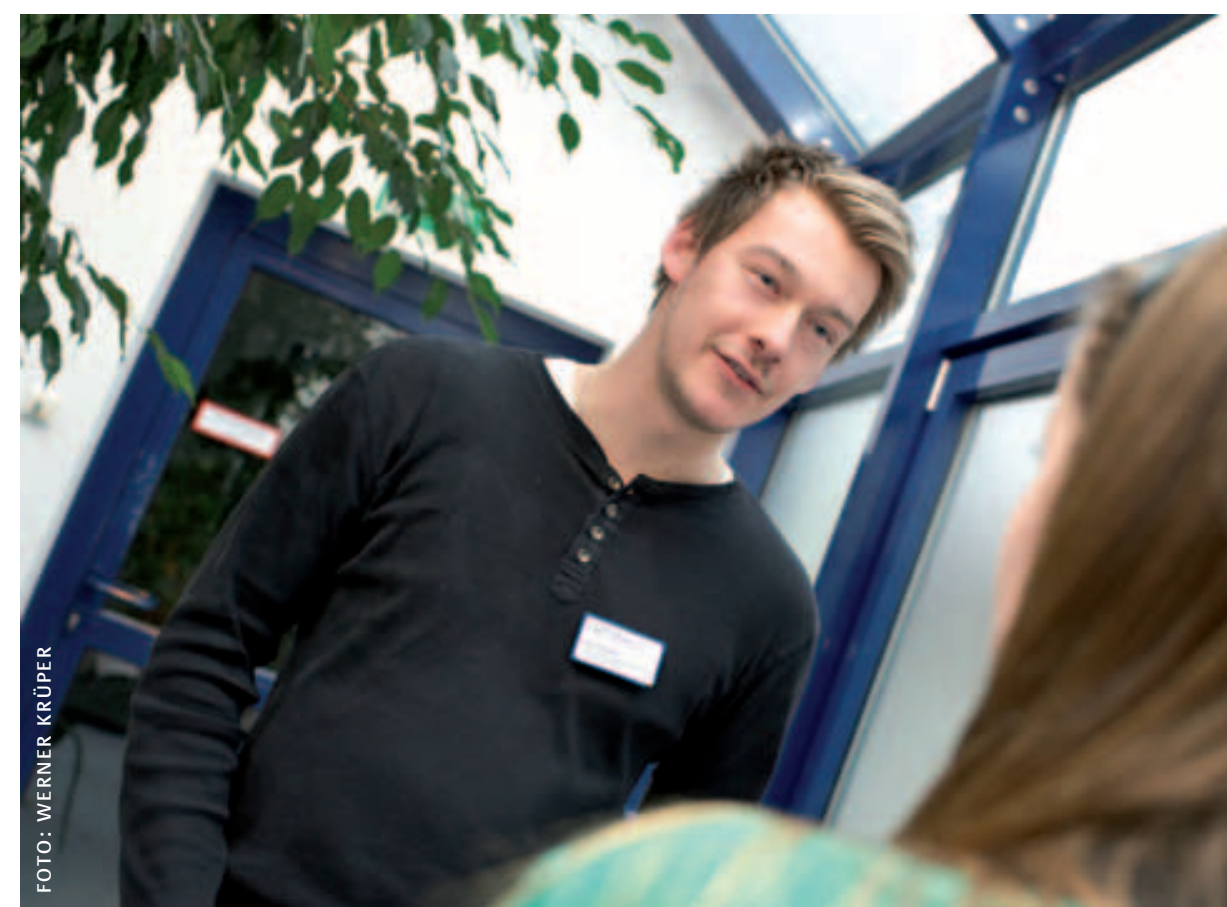

Ähnlich diffus waren im Beobachtungszeitraum auch die Wir-Botschaften, also die (meist indirekten) Aussagen über die Beziehung zwischen den Kommunikationspartnern. Wer an stationäre Psychiatrie denkt, hat meist eine extreme Hierarchie zwischen den Behandlern (als den Mächtigen) und den Patienten (als den Ohnmächtigen) vor Augen. Die Quasi-TUB ergab jedoch ein differenzierteres Bild. Bei der Depressions- und Krisenstation handelte es sich um eine halboffene Station, d. h. die Station konnte in der Regel frei betreten und verlassen werden. Generell erschien der Umgang mit „freiheitsentziehenden Maßnahmen“ recht behutsam: Die Mitarbeiter waren stets bemüht, auf die Ausübung von Zwang und Gewalt zu verzichten und stattdessen Absprachen mit den Patienten zu treffen.

Allerdings stellt Ingrid Darmann [6] mit Recht fest, dass es in der Krankenpflege neben „zwingender Macht“ auch eine „verweigernde Macht“ gibt. Die Quasi-TUB ergab den Eindruck, dass die Beziehung zwischen Mitarbeitern und Patienten gerade durch die ,verweigernde Macht“ stark geprägt wurde. Denn im Depressionsund Krisenbereich war die Klinik für viele Patienten die letzte Anlaufstelle in größter persönlicher Not. Dieses Angewiesensein auf Hilfe und das Wissen, nirgendwo anders hinzukönnen, führten zu einer besonderen Art von Ohnmacht. Die Behandler erhielten dadurch die Möglichkeit, Macht auf eine subtile Weise auch ohne äußeren Zwang auszuüben. Dies geschah wiederholt, indem Patienten vor die Wahl gestellt wurden, entweder den „Vorschlägen“ des Behandlungskonzeptes zu folgen oder nach Hause zu gehen. Konkret konnte das im 
Einzelfall heißen: „Wenn du weiterleben willst, dann nur unter unseren Bedingungen!“

Ein anderer zentraler Aspekt des Themas „Beziehungsschemata“ betrifft die kollektive Beziehung zwischen Mitarbeitern und Patienten. Schon durch die Anredeformen entstanden quasi Blöcke, denn die Mitarbeiter (einschließlich Praktikanten und Pflegeschüler) duzten sich grundsätzlich untereinander, sprachen die Patienten aber mit „Sie“ an. Das wäre kaum bemerkenswert, wenn nicht die Nähe zwischen den Mitarbeitern und ihre Distanz gegenüber den Patienten auch in anderen Dingen ihren Ausdruck gefunden hätten. So hatten einige Mitarbeiter die Angewohnheit, in der Wir-Form zu sprechen, wenn es um Behandlungsfragen ging. Dem lag der Gedanke zugrunde, dass alle mit einer Patientin befassten Mitarbeiter ein „Team“ bildeten. Folglich wurde oft vom „Behandlungsteam“ gesprochen und es schien selbstverständlich zu sein, dass der Patient diesem Team nicht angehörte. Er war lediglich Objekt der Behandlung durch die professionellen Kräfte. Diese Beziehung manifestierte sich insofern in der Kommunikation, als der weitaus größte Teil der Gespräche über die Patienten geführt wurde, nicht mit ihnen. Die regelmäßigen Teambesprechungen der Mitarbeiter fanden teils in einsehbaren Räumen statt, sodass die Patienten von außen zusehen konnten, wie über sie verhandelt wurde - zuhören und mitreden durften sie nicht.

Dem Eindruck, dass die Patienten alleine einem anonymen Behandlungssystem ausgeliefert waren, wirkten jedoch einzelne Mitarbeiter nach Kräften entgegen. Wir haben oben im Zusammenhang mit dem Schema des fokussierten Interagierens bereits betont, dass es auch sehr persönliche Gespräche zwischen Mitarbeitern und Patienten gab, in denen die Rollenzuschreibungen des Systems „Psychiatrie“ in den Hintergrund rückten. Durch beidseitige Offenheit und das ehrliche Interesse am Gegenüber entstand eine persönliche Beziehung, die für manche Patienten heilsamer war als sämtliche vom Behandler-Team erdachten Maßnahmen. Von Bedeutung waren dabei nicht nur die
Gesprächsinhalte und die Kommunikation auf Augenhöhe. Vielmehr wirkten auch nonverbale Anteile wie ein fester Händedruck oder eine Umarmung unmittelbar beruhigend und ermutigend.

Problematisch für Mitarbeiter und Patienten gleichermaßen war jedoch, dass es in der Klinik keine erkennbar einheitliche Haltung zu solchen Botschaften auf der Beziehungsebene gab. Ein Teil der Mitarbeiter bemühte sich immer wieder darum, Patienten mit menschlicher Anteilnahme zu begegnen; die Pflegewissenschaft spricht in diesem Zusammenhang von Empathie. Für das Selbstbild der Patienten bedeutete dies, dass sie sich nicht mehr als unerträglich empfinden mussten, sondern erfahren durften, dass andere Menschen es mit ihnen aushalten konnten - auch in einer Krise. Genau diesen, ungeheuer befreienden und heilsamen Effekt hielt ein anderer Teil der Mitarbeiter jedoch für fatal. Sie sahen Depressionen anscheinend vor allem als chemisch-kognitive Störung, der mit Medikamenten (chemisch) sowie einem Prozess des Umlernens (kognitiv) zu begegnen sei. Und wie oben bereits erläutert, lehnt die lerntheoretisch fundierte Verhaltenstherapie es ab, auf Krisensituationen mit menschlicher Zuwendung zu reagieren, weil dadurch „unerwünschtes Verhalten“ belohnt und verstärkt würde. Deshalb mussten sich diejenigen Mitarbeiter, die Patienten in Krisen mit menschlicher Nähe begegneten, vor Kollegen für ihr angeblich unprofessionelles, distanzloses Verhalten rechtfertigen. Das wiederum war auch eine Belastung für die Patienten, die gerade die für sie hilfreichsten Mitarbeiter nicht in Schwierigkeiten bringen wollten.

\section{Was folgt daraus? Weiterführende Überlegungen}

Die Quasi-TUB erbrachte eine Vielzahl teils heterogener Eindrücke. Zwei Charakteristika prägten jedoch weite Teile der betrachteten Kommunikation: Erstens zeigte sich eine hierarchische Strukturierung; die Patienten fanden sich über die einzelnen Kontextualisierungsschemata hinweg meist in der Rolle der nur Reagierenden. Zweitens fiel insbesondere bei den Beziehungsschemata auf, dass widersprüchliche (inkongruente) Botschaften vermittelt wurden. Diese beiden Punkte und ihre Konsequenzen möchten wir im Folgenden näher in den Blick nehmen.

\section{Hierarchische Kommunikation}

Die Situationen, die im Beobachtungszeitraum durch Kommunikation geschaffen bzw. aufrechterhalten wurden, beinhalteten häufig ein Ungleichgewicht. Mit Blick auf die einzelnen Kontextualisierungsschemata lässt sich dies wie folgt zusammenfassen:

\section{Fokussiertes Interagieren}

Meist waren es die Mitarbeiter, die Gespräche mit den Patienten begannen und beendeten sowie über die räumliche und zeitliche Verortung entschieden. Letztere bestimmte wiederum darüber, inwiefern gegenseitige Aufmerksamkeit überhaupt möglich war.

\section{Turn taking}

Auch die innere Strukturierung der Kommunikation oblag in weiten Teilen den Behandlern, insbesondere dann, wenn standardisierte Gesprächsleitfäden befolgt oder diverse Fragebögen ausgewertet wurden.

\section{Handlungsschemata}

Über die Handlungsabläufe bestimmten vor allem die Routinen des Klinikalltags, an die der Patient sich anzupassen hatte. Patienten wurden dadurch weitgehend $\mathrm{zu}$ Objekten einer (Be-)Handlung. Zugleich war jedoch auch immer wieder ehrliches Bemühen um individuelle Lösungen erkennbar.

\section{Thematische Schemata}

Die Wahl der thematischen Schwerpunkte war wesentlich von der (verhaltens-) therapeutischen Konzeption der Station bestimmt. Dies führte dazu, dass Patienten daran gehindert wurden, allzu ausführlich über ihre Lebensgeschichte und ihre individuellen Wertvorstellungen zu sprechen. Von Interesse war nur das „Hier und Jetzt“ und dort wiederum das Denken, Fühlen und Handeln des Patienten, soweit es krankheitsbezogen war und sich leicht in Tabellenform erfassen ließ. 


\section{Beziehungsschemata}

Die Beziehung zwischen Mitarbeitern und Patienten erschien komplex. Einerseits war in vielen expliziten Botschaften das Bemühen um eine Selbstwertstärkung der Patienten erkennbar; andererseits trug die gesamte Rahmung der Kommunikation ständig zu einer Selbstwertschwächung bei.

Auch wenn es sich bei diesen Aussagen nur um den Befund einer (allerdings

längerfristigen und theoretisch unterfütterten) Einzelfallbeobachtung handelt, können die Ergebnisse nicht als befriedigend bezeichnet werden. Tatsächlich

Auch nonverbale Anteile der Kommunikation wie ein fester Händedruck oder eine Umarmung wirkten unmittelbar beruhigend und ermutigend.

bleibt aber die Frage, warum die Botschaften gerade auf der Beziehungsebene oft so widersprüchlich waren. Wir vermuten, dass unter den Behandlern kein Konsens über ihr Menschenbild und über ihre grundsätzliche Einstellung gegenüber den Patienten bestand; schlimmer noch: dass zumindest ein Teil von ihnen noch nie intensiv über solche Haltungsfragen nachgedacht hatte. Stattdessen wurden schlicht die vorgegebenen therapeutischen Konzepte umgesetzt. Und diejenigen unter den Mitarbeitern, die einmal bewusst einen helfenden Beruf ergriffen hatten, bemühten sich nach

Menschen ausmachte - umprogrammieren ließ, um wieder zu einem funktionsfähigen Mitglied der Gesellschaft zu werden. Diese Inkongruenz zwischen angeblicher Annahme und tatsächlicher Abwertung der Patienten, zwischen angeblicher Ressourcenorientierung und tatsächlicher Nutzung individueller Fähigkeiten nur zur Beseitigung diagnostizierter Defizite war für sich alleine genommen schon verwirrend genug. Noch komplexer wurde das Gesamtbild dadurch, dass ein Teil der Mitarbeiter sich im Gegensatz dazu nachdrücklich um Kongruenz bemühte. Wir haben oben im Zusammenhang mit den einzelnen Kontextualisierungsschemata darauf hingewiesen, dass es immer wieder auch persönliche Kommunikation zwischen Mitarbeitern und Patienten gab, die von ehrlichem Interesse und menschlicher Zuwendung geprägt war. Hier wurde die Botschaft „Du bist okay“ ohne Einschränkungen und Bedingungen zum Ausdruck gebracht, und zwar nicht durch ständig wiederholte Lehrbuchsätze, sondern implizit durch die Art des Umgangs mit dem einzelnen Menschen. Andererseits waren aber auch diese Mitarbeiter an anderer Stelle in therapeutische Maßnahmen involviert, die von Patienten als demütigend und erniedrigend empfunden wurden, beispielsweise im Rahmen von verhaltenstherapeutischen Bestrafungen.

Dass derartige Diskrepanzen für viele Patienten verunsichernd und belastend waren, wird kaum überraschen. Offen
Kräften, nebenbei noch etwas Menschlichkeit einzubringen.

Eine humane, den Patienten wirklich wertschätzende Psychiatrie wäre vielleicht eher erreichbar, wenn sich alle Mitarbeiter ehrlich einer Reihe von Grundsatzfragen stellen würden:

1. Gibt es ein Recht auf Krankheit? Das bedeutet: Werden Lebenskrisen als Zeichen des Versagens in einer allein auf Leistung ausgerichteten Gesellschaft gesehen oder als Möglichkeit zum persönlichen Wachstum?

2. Was ist das oberste Ziel psychiatrischer Behandlung: die Wiederherstellung der gesellschaftlichen Funktionsfähigkeit des Patienten oder dessen subjektiv empfundene Genesung?

3. Welches ist der angemessene Weg zur Gesundung bzw. Genesung: Sollen die Behandler den Patienten zu erwünschtem Verhalten erziehen? Oder besteht die gemeinsame Aufgabe von Patienten und Behandlern darin, nach individuellen Wegen des Wachstums zu suchen und die Selbstheilungskräfte zu aktivieren? Kurz: Geht es ums Zurechtstutzen oder darum, Raum, Licht und Luft zum Wachstum zu geben?

4. Wenn die gesellschaftlichen Bedingungen immer mehr Menschen psychisch krank werden lassen: Ist dann die Psychiatrie der verlängerte Arm der Gesellschaft, um die Menschen wieder funktionsfähig zu machen (oder auszusortieren)? Oder ist die Psychiatrie der Anwalt der Betroffenen, ein Unterstütwertgefühls ab. Implizit drückte die Kommunikation hingegen nicht selten das ge- 
zer im Ringen um eine lebensgerechtere Welt?

Inkongruenz entsteht dadurch, dass die Psychiatrie sich in der Praxis um die klare Beantwortung dieser Fragen bislang weitgehend herumdrückt. Stattdessen wird versucht, ökonomisch-funktionalistisches Denken und humane Restwerte irgendwie miteinander zu vereinbaren. Leidtragende dieses faulen Kompromisses sind die Patienten. Leidtragende sind auch diejenigen Mitarbeiter, die sich ehrlich um Empathie und persönliche Zuwendung bemühen. Es wäre zu wünschen, dass Patienten und engagierte Mitarbeiter gemeinsam für eine Psychiatrie eintreten, in der eine menschliche, persönliche, gegenseitig wertschätzende und damit heilsame Kommunikation selbstverständlich ist.

\section{LITERATUR}

1 Auer, P. Kontextualisierung. Studium Linguistik 1986; 19: 22-47

2 Auer, P. Introduction: John Gumperz Approach to Contextualization. In: Auer, P., Di Luzio, A. (Hrsg). The Contextualization of Language. Amsterdam: John Benjamins, 1992: 1-38

3 Bartlett, F.C. Remembering: A Study in Experimental and Social Psychology. Cambridge: Cambridge University Press, 1932 4 Buchanan-Barker, P., Barker, P.J. Eine Klärung der grundlegenden Werte von Recovery: Die 10 Tidal Verpflichtungen. Deutsche Übersetzung von Ruth Schröck, bearbeitet von Michael Schulz, Ian Needham, Christoph Abderhalden. Zeitschrift für Pflegewissenschaft und psychische Gesundheit 2008; 2(1): 12-22

5 Cook-Gumperz, J., Gumperz, J.J. Context in Children's Speech. Papers on Language and Context. Berkeley: Univ. of California, Language Behavior Research Laboratory, 1976 (Working Paper 46)

6 Darmann, I. Kommunikative Kompetenz in der Pflege. Ein pflegedidaktisches Konzept auf der Basis einer qualitativen Analyse der pflegerischen Kommunikation. Stuttgart: Kohlhammer, 2000
7 Erdwien, B. Kommunikationsstrukturen in der Arzt-Patient- und Pflege-Patient-Beziehung im Krankenhaus: Empirische Untersuchungen zur Patientenzufriedenheit unter Berücksichtigung der subjektiven Erlebnisperspektive von Patienten, Ärzten und Pflegepersonal. Berlin: dissertation. de, 2005

8 Girtler, R. Die „teilnehmende unstrukturierte Beobachtung“ - ihr Vorteil bei der Erforschung des sozialen Handelns und des in ihm enthaltenen Sinns. In: Aster,

R. / Merkens, H., Repp, M. (Hrsg). Teilnehmende Beobachtung. Werkstattberichte und methodologische Reflexionen. Frankfurt: Campus, 1989: 103-113

9 Goffman, E. Rahmen-Analyse. Ein Versuch über die Organisation von Alltagserfahrungen. Übersetzt von Hermann Vetter. Frankfurt a. M.: Suhrkamp, 1977

10 Goffman, E. Behavior in Public Places. New York: Free Press, 1963

11 Gumperz, J.J. Contextualization Revisited. In: Auer, P., Di Luzio, A. (Hrsg). The Contextualization of Language. Amsterdam / Philadelphia: Benjamins, 1992: 39-53

12 Gumperz, J. Discourse Strategies. Cambridge: C.U.P., 1982

13 Hymes, D. Soziolinguistik. Zur Ethnographie der Kommunikation. Eingeleitet und herausgegeben von Florian Coulmas. Frankfurt a. M.: Suhrkamp, 1979

14 Körfer, A., Köhle, K., Obliers, R. Zur Evaluation der Arzt-Patienten-Kommunikation - Perspektiven einer angewandten Diskursethik in der Medizin. In: Redder,

A., Wiese, I. (Hrsg). Medizinische Kommunikation: Diskurspraxis, Diskursethik, Diskursanalyse. Opladen: Westdeutscher Verlag, 1994: 53-94

15 Lefrancois, G. R. Psychologie des Lernens. Vierte, überarbeitete und erweiterte Auflage. Heidelberg: Springer, 2006

16 Quasthoff, U. Kommunikative Normen im Entstehen: Beobachtungen zu Kontextualisierungsprozessen in elektronischer Kommunikation. In: Weingarten, R. (Hrsg). Sprachwandel durch Computer. Opladen: Westdeutscher Verlag, 1997: 23-50
17 Quasthoff, U. Das Prinzip des primären Sprechers, das Zuständigkeitsprinzip und das Verantwortungsprinzip. Zum Verhältnis von „Alltag“ und „Institution“ am Beispiel der Verteilung des Rederechts in der Arzt-Patienten-Interaktion. In: Ehlich, K. et al. (Hrsg). Medizinische und therapeutische Kommunikation. Diskursanalytische Untersuchungen. Opladen: Westdeutscher Verlag, 1990: 66-81

18 Schädle-Deininger, H. Pflegerische Sprache - Spiegelbild der Wirklichkeit? Psych Pflege 2006; 12: 181-182

19 Schulz von Thun, F. Miteinander reden. Band 1. Störungen und Klärungen. Reinbek: Rowohlt, 1981

20 Skinner, B.F. Beyond freedom and dignity. New York: Knopf, 1971

21 Skinner, B.F. Contingencies of reinforcement: A theoretical analysis. New York: Appleton-Century-Croft, 1969 22 Watzlawick, P., Beavin, J.H., Jackson, D.D. Menschliche Kommunikation. Formen, Störungen, Paradoxien. Bern: Verlag Hans Huber, 1974

23 Weingarten, R. Reformulierungen in der Gesprächspsychotherapie. In: Ehlich, K. et al. (Hrsg.), Medizinische und therapeutische Kommunikation. Diskursanalytische Untersuchungen. Opladen: Westdeutscher Verlag, 1990: 228-240

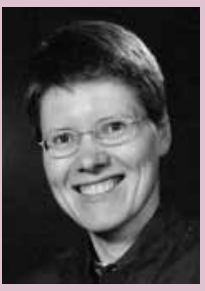

Dr. Elke Prestin Studium der Linguistik, Anglistik und Soziologie (M.A.), anschließend Promotion im Fach Linguistik (Dr. phil.), Ausbildung und mehrjährige Berufstätigkeit als Redakteurin, Tätigkeiten in Forschung und Lehre an der Universität Bielefeld, der Universität Greifswald, der FH Ostwestfalen-Lippe und der FH Darmstadt.

Kontaktadresse:

Dr. Elke Prestin

E-Mail: Prestin@web.de

Bibliografie

DOI 10.1055/s-0031-1275380

Psych Pflege 2011; 17: 87-98

(c) Georg Thieme Verlag KG

Stuttgart · New York · ISSN 0949-1619 